\title{
On two effects of signaling the consequences for remembering
}

\author{
B. MAXWELL JONES \\ University of Auckland, Auckland, New Zealand \\ and \\ K. GEOFFREY WHITE and BRENT L. ALSOP \\ University of Otago, Dunedin, New Zealand
}

\begin{abstract}
Five pigeons were trained to perform a delayed matching-to-sample task in which red- and greencolored keys were presented as sample and choice stimuli, and the duration of a delay interval varied across trials. Experiment 1 investigated the effects on delayed-matching accuracy of signaling different durations of food access for the two correct responses (the differential-outcomes effect), and of signaling nondifferential but larger durations for both responses (the signaled-magnitudes effect). In Condition 1, a vertical bar on either sample signaled different rewards (or different outcomes, DOs) for correct red and correct green responses ( 0.5 and $3.5 \mathrm{sec}$, respectively), and a horizontal bar signaled equal durations of food access (or same outcomes, SOs) for these responses $(1.5 \mathrm{sec}$ ). In Condition 2, the horizontal bar signaled equally large rewards for the two correct responses ( $3.5 \mathrm{sec}$ ), and the vertical bar signaled equally small rewards $(0.5 \mathrm{sec})$. Delayed-matching accuracies were higher on DO trials than on SO trials, and they were higher on large-reward trials than on smallreward trials. However, analyses of discriminability estimates as a function of delay-interval duration revealed differences between the forgetting functions reflecting these two effects. Signaling DOs increased the initial level of the function and reduced its slope relative to signaling SOs, whereas signaling larger rewards increased the initial level of the function but did not affect its slope relative to signaling smaller rewards. Experiment 2 investigated whether the difference between the initial levels of DO and SO functions in Condition 1 resulted from overall longer food access on the former trials. However, varying the food-access times on SO trials across three conditions $(0.5,3.5$, and $1.5 \mathrm{sec}$ ) failed to produce systematic effects consistent with this hypothesis. The results are discussed with respect to the mechanisms that could be responsible for the two effects.
\end{abstract}

Laboratory experiments investigating the short-term memory abilities of nonhuman animals typically examine the behavior of their subjects in some type of delayed conditional discrimination. These procedures are characterized by discrete trials involving five distinct phases: the presentation of a sample (or to-be-remembered) stimulus, a delay interval spent in darkness, a choice phase providing an opportunity to respond in accordance with the sample, stimulus consequences of the choice response, and an intertrial interval (ITI). For example, in the prototypical delayed matching-to-sample (DMTS) task, a trial begins with the presentation of either a redor a green-colored field on the center response key (the

This research was supported by grants from the New Zealand University Grants Committee to K.G.W. and by an N.Z.V.C.C. Postgraduate Scholarship to B.M.J. We are grateful to Barry Dingwall for technical assistance, to Neroli Harris for care of the birds, and to Lewis Bizo and James Hegarty for help with running the experiments. During the preparation of this manuscript, Michael Davison and staff at the Experimental Analysis of Behaviour Research Unit at the University of Auckland provided many valuable discussions. Reprints may be obtained from B. M. Jones, Department of Psychology, University of Auckland, Private Bag 92019, Auckland, New Zealand (e-mail: bmjones@auckland.ac.nz). sample stimulus). Following some number of responses to this stimulus, the chamber is darkened and a delay interval of some variable duration elapses. At the end of this interval, the two side keys are illuminated either red on the left and green on the right or vice-versa. A response to the side key presenting the color that matches the most recently presented sample is reinforced with access to food; a response to the nonmatching side key goes unreinforced and produces an equivalent period of chamber darkness. An ITI, usually of a fixed duration, then elapses before the next sample stimulus is presented. The order in which the two samples are presented, the position of the correct comparison stimulus, and the duration of the delay interval are generally determined randomly so that all vary across trials in an unpredictable manner.

To date, most experimental analyses of remembering in nonhumans have focused on the effects of stimulus conditions prior to the choice response (see McCarthy \& Nevin, 1991). In contrast, relatively few studies have investigated the effects that the consequences for remembering have on behavior in DMTS tasks.

An emphasis on the effects of conditions preceding the choice response appears to reflect an assumption 
regarding the functional role of rewards in DMTSnamely, that the stimulus conditions following choice responses (e.g., food access for correct responses and chamber darkness for incorrect responses) do not affect delayed-matching accuracy directly, but rather affect accuracy only by facilitating learning of the stimulusresponse contingencies that define the conditional discrimination (e.g., Honig, 1978). For example, Peterson and Trapold (1980) suggest that the commonly accepted theoretical account of the function of rewards in conditional discriminations (delayed and otherwise) is based on Thorndike's (1898) "law of effect" and Skinner's (1938) principle of reinforcement. According to this view, the rewards for correct choice responses strengthen or reinforce the "stimulus-response association," or the "stimulus control" between the sample stimuli and their respective correct responses, and the exact identity of the rewards in the task is unimportant. However, several recent findings suggest that the exact identity of the rewards in a DMTS task can be important. The present research examined two phenomena revealed in manipulations of the consequences (rewards) for remembering: the differential-outcomes effect (DOE) and the signaledmagnitude effect (SME).

In research concerned with nonhuman short-term memory, the DOE refers to more accurate delayed matching when different rewards (or different outcomes, DOs) follow the two correct responses than when the same rewards (or same outcomes, SOs) are used (Brodigan \& Peterson, 1976). The DOE has been demonstrated with a range of trial outcomes in between-group and betweencondition experimental designs involving a range of delayed-conditional discriminations. For example, relative to an SO group or condition, or a control group receiving both outcomes but uncorrelated with the samples, delayed matching is more accurate when different qualities of reward (Brodigan \& Peterson, 1976), different probabilities of food presentation (DeLong \& Wasserman, 1981; Santi \& Roberts, 1985a, 1985b; Urcuioli, 1990a, 1990b), different locations of a food dispenser (Williams, Butler, \& Overmier, 1990), and even food versus no food (Linwick, Overmier, Peterson, \& Mertens, 1988; Peterson, Linwick, \& Overmier, 1987; Peterson, Wheeler, \& Trapold, 1980; Urcuioli, 1990b) have been correlated with the two samples and two correct responses.

The SME, on the other hand, refers to more accurate matching in a delayed-conditional discrimination when larger magnitudes of a reward are arranged for both correct responses than when smaller magnitudes are arranged. The majority of studies investigating this phenomenon have been conducted with humans (see Nelson, 1976, for a review). For example, Eysenck and Eysenck (1982) showed that words printed in a color signaling a large monetary reward for later remembering were recalled more accurately than were words printed in a color signaling a smaller reward. However, an investigation of the SME with nonhumans was conducted by Nevin and Grosch (1990). In this study, pigeons were trained in a DMTS task with red-and green-sample and comparison stimuli, and a range of delay intervals varied within sessions. On a random half of the trials, an auditory stimulus ( $750-\mathrm{Hz}$ tone) was presented in conjunction with either sample and throughout the delay interval to signal that both correct choice responses would earn a large reward (4.5-sec access to food). On the other half of the trials, a different auditory stimulus (white noise) signaled that both correct choice responses would earn a smaller reward (1.5-sec access to food). Nevin and Grosch found that, for all birds, matching accuracy at each delay interval was higher on the large-reward trials than on the small-reward trials.

Although arranging DOs and signaling larger rewards both enhance delayed-matching accuracy, it is possible that the two manipulations have different effects on delay-interval functions. For example, numerous studies investigating the DOE have reported that the facilitative effect of DOs on percentage correct scores increases as the delay interval increases (e.g., Brodigan \& Peterson, 1987; DeLong \& Wasserman, 1981; Peterson, Wheeler, \& Armstrong, 1978; Peterson et al., 1980). In other words, arranging DOs appears to attenuate the detrimental effect that increasing delay intervals have on delayed-matching accuracy. In contrast, Nevin and Grosch (1990) reported that the delay-interval functions describing accuracies on large-reward and small-reward trials were similar in slope and differed only in terms of their initial level (see their Figure 1). Possible differences between the functions characterizing the DOE and the SME are theoretically interesting because different processes have frequently been associated with the initial level of delay-interval functions and their slope. For instance, in recent behavioral models of DMTS performance by nonhumans (e.g., Harnett, McCarthy, \& Davison, 1984; White \& McKenzie, 1982), the initial level of the delay-interval function has been identified with time-independent processes determining the initial discriminability between the samples, and the slope of the function has been identified with time-dependent processes determining rate of forgetting (McCarthy \& White, 1987; Nevin \& Grosch, 1990; White, 1985; 1991).

However, empirical evidence for different quantitative effects of signaling DOs and signaling larger rewards is severely limited by the fact that this evidence rests with comparisons across studies. These studies have involved markedly different procedures using a range of sample stimuli, comparison stimuli, and reward dimensions, so that direct comparisons are difficult. Most notably, studies investigating the DOE have always arranged DOs for one group and not another, or in one condition but not another, so that the specific reward on each trial is signaled by the sample stimulus. In contrast, to the best of our knowledge, no such between-group or between-condition designs have been employed in studies of the SME (see Nelson, 1976, for an explanation). These studies have all varied the magnitude of rewards 


\section{Different-Outcome Trials}

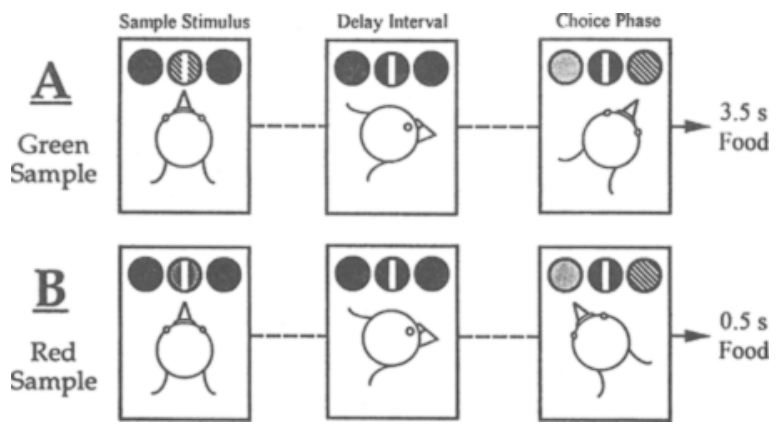

Same-Outcome Trials

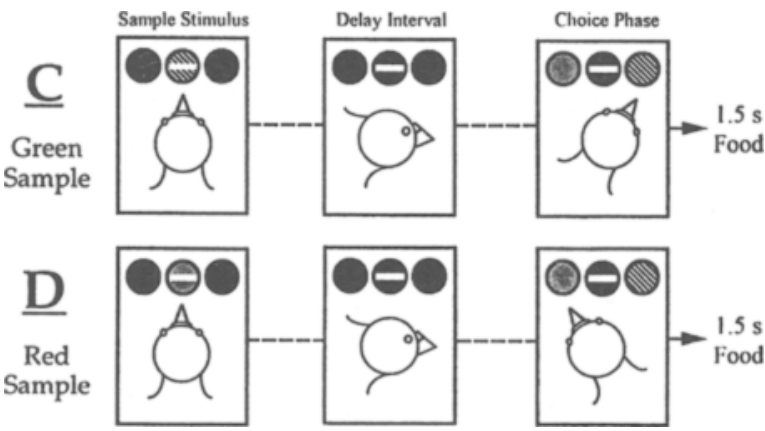

Figure 1. Stimulus arrangements and sequence of events defining the four trial types in Condition 1 of Experiment 1. Trials began with the presentation of the green or the red sample stimulus, and the vertical-bar or horizontal-bar outcome cue on the center key. Following five responses to this stimulus, the sample extinguished (but the outcome cue remained), and a delay interval of $0.01,1,4$, or $8 \mathrm{sec}$ elapsed before the side keys were lit green on the left and red on the right, or vice versa, for the choice phase. On different-outcome trials (signaled by the vertical bar), correct green responses (A) produced 3.5-sec access to wheat, whereas correct red responses (B) produced 0.5-sec access to wheat. On same-outcome trials (signaled by the horizontal bar), both correct red and correct green responses were followed by the same duration of food access ( $C$ and $D)$.

within sessions and have signaled the specific reward on each trial by presenting an "outcome cue" in conjunction with the sample stimuli. The effects of such procedural differences on characteristics of delay-interval functions are extremely difficult to predict. The present experiment therefore arranged a DMTS procedure that permitted a within-subject comparison of the DOE and the SME. This was conducted by a within-session study of each phenomenon. Red- and green-sample and comparison stimuli were used, and the duration of the delay interval varied within sessions. One condition investigated the DOE. In this condition, on a random half of the trials, a vertical bar was superimposed on the sample stimuli, and different durations of food access (DOs) followed the two correct responses (correct-red and correct-green responses). On the remaining trials, a horizontal bar appeared on the sample, and both correct re- sponses produced the same duration of food access (SOs). A second condition investigated the SME. Here, the vertical bar on either sample signaled that the same but relatively short durations of food access would follow both correct responses (small-reward trials), whereas the horizontal bar signaled that longer durations of food access would follow both correct responses (large-reward trials).

A second factor limiting the reliability of quantitative differences between the DOE and the SME involves mathematical properties of the dependent measures used in many of these studies. In particular, studies investigating the DOE have almost invariably measured delayedmatching accuracy in terms of the proportion of trials on which the correct comparison stimulus was chosen (proportion correct, or PC). However, as several authors have noted (McCarthy \& White, 1987; Nevin \& Grosch, 1990 ), $P C$ is a bounded and biased measure of discrimination accuracy so that comparisons of changes in PC are difficult to interpret at different accuracy levels. Furthermore, qualitatively or quantitatively different rewards in a DO procedure are likely to produce significant biases in choice responding (at least relative to an SO group or condition), and the degree of this bias may vary across delay intervals (see Jones \& White, 1992; McCarthy \& Davison, 1991). Therefore, PC would seem to be an inappropriate measure on which to base analyses of quantitative differences between the DOE and the SME. Consequently, the present study analyzed delayinterval functions in terms of a quantitative description of DMTS performance (White \& McKenzie, 1982), which uses an index of discrimination accuracy $\left(\log d_{t}\right)$ that is unbounded and theoretically bias-free. White and McKenzie's analysis also explicitly offers parameters that measure the initial level and the slope of delayinterval functions.

\section{EXPERIMENT 1}

\section{Method}

\section{Subjects}

Five adult homing pigeons, labeled D1 through D5, were maintained at $85 \%$ of their free-feeding body weights by supplementary feeding of mixed grain following experimental sessions. Water and grit were freely available in the home cages. Experimental ses sions were conducted daily for each bird, provided its weight was not greater than $15 \mathrm{~g}$ above the target $85 \%$ weight. All of the birds had prior experience with DMTS procedures.

\section{Apparatus}

A lightproof, sound-attenuating experimental chamber $(33 \mathrm{~cm}$ wide, $33 \mathrm{~cm}$ deep, and $34 \mathrm{~cm}$ high) contained an interface panel and an exhaust fan. Three horizontally aligned translucent response keys were mounted $25.6 \mathrm{~cm}$ above a wire grid floor on the interface panel. The keys were $2.85 \mathrm{~cm}$ in diameter and were set $9.15 \mathrm{~cm}$ apart, center to center. A minimum force of $0.15 \mathrm{~N}$ operated a microswitch mounted behind each key.

The two side keys could be illuminated red or green by lamps situated behind them. An in-line stimulus projector (IEE with 24-V \#1820 lamps) was mounted behind the center response key. This projector contained Kodak Wratten filters ( $72 \mathrm{~B}$ and 74 ) that 
could illuminate the rear surface of the key with either red $(606 \mathrm{~nm}$ peak wavelength) or green ( $538 \mathrm{~nm}$ peak wavelength) colored fields. These stimuli were not equated for brightness. A white horizontal bar and a white vertical bar could also be presented on the center key against either the background of the sample stimulus or a black background. Each bar was $0.92 \mathrm{~cm}$ wide and covered the diameter of the key. These stimuli were projected with white (unfiltered) light. Effective responses to the illuminated center key were followed by a 50-msec offset of the key stimulus.

A hopper opening $(5.5 \mathrm{~cm}$ wide $\times 6.5 \mathrm{~cm}$ high) was located directly below the center key and $5.3 \mathrm{~cm}$ from the floor. A solenoidoperated hopper (Campden Instruments) delivered wheat through this opening. Presentations of the hopper were accompanied by white light illumination of the aperture and the extinction of all key stimuli. An infrared-emitting diode and a phototransistor were mounted opposite each other inside the hopper opening. The infrared beam detected when the bird's head was in the hopper opening during hopper presentation, and food access was timed from this moment.

All experimental events were controlled and recorded by a Digital PDP 11/23 microcomputer located in an adjoining room. This computer ran time-shared SKED-11 software.

\section{Procedure}

Preliminary training. Prior to this experiment, all of the birds had been performing a variant of DMTS in which the sample stimuli were hopper presentations versus hopper illumination and the comparison stimuli were red and green side keys. Because of this prior experience, only minimal preliminary training was required before the first experimental condition began.

In each session of preliminary training, each of 128 discrete trials began with red or green illumination of the center key. The fifth peck to this key (FR 5) darkened it and immediately lit one of the side keys with the color that matched the center-key color. A response to the lit side key darkened it and produced 2-sec access to food followed by a 10-sec ITI of chamber darkness. A quasi-random order of key colors and left or right side-key presentations was arranged in each session. Each bird received a minimum of 10 sessions of this "forced-choice" training.

Experimental conditions. All birds received a minimum of 60 sessions training in both experimental conditions. Condition 2 did not immediately follow Condition 1 but was separated by three further conditions described in Experiment 2.

Figure 1 illustrates the sequence of events that defined the four trial types in Condition 1. Each trial began with the presentation of either the red or the green sample stimulus and the vertical-bar or the horizontal-bar outcome cue on the center key. Five responses to this key (FR 5) extinguished only the sample (the outcome cue remained) and initiated a delay interval of $0.01,1,4$, or $8 \mathrm{sec}$. Following the delay interval, the two side keys were lit red on the left and green on the right, or vice versa, for the choice phase. A single response to either side key extinguished all key stimuli. A response to the side key with the color that matched the sample color was deemed correct and reinforced with either a large or a small reward depending on which outcome cue had appeared. On trials where the outcome cue was the vertical bar (DO trials), correct responses following the green sample produced 3.5 -sec access to wheat, whereas correct responses following the red sample produced only $0.5-\mathrm{sec}$ access to wheat. On trials where the outcome cue was the horizontal bar (SO trials), both correct red and correct green responses were followed by the same $(1.5 \mathrm{sec})$ duration of food access. An incorrect response produced a 3-sec chamber blackout, and following either food access or blackout, there was a dark ITI of $10 \mathrm{sec}$ before the next trial began.

To the best of our knowledge, no previous research has investigated how nonhumans average reinforcer durations. Thus, it was impossible to calculate what duration of food access on SO trials might be hedonically equivalent to the two durations ( 3.5 and $0.5 \mathrm{sec}$ ) arranged on DO trials. Our choice of reinforcer duration on SO trials was therefore somewhat arbitrary, although it was approximately intermediate between the arithmetic and geometric means of the durations of food access on DO trials ( 2 and $1.32 \mathrm{sec}$, respectively). (Experiment 2 explicitly investigated the effects on matching accuracies of the food-access duration arranged on SO trials.)

Figure 2 illustrates the sequence of events that defined the four trial types in Condition 2. These trials were identical to those arranged in Condition 1, except that different food-access durations now followed the four sample-outcome cue compounds. Shorter but equal durations of food access for both correct responses $(0.5 \mathrm{sec}$ on small-reward trials) were signaled by superimposing the vertical bar over either sample, and longer durations of food access for both correct responses ( $3.5 \mathrm{sec}$ on large-reward trials) were signaled by the horizontal bar. Thus, the outcome cue that was associated with different rewards in Condition 1 now signaled the smaller reward, and the outcome cue that was earlier associated with same rewards now signaled the larger reward. This

\section{Small-Reward Trials}

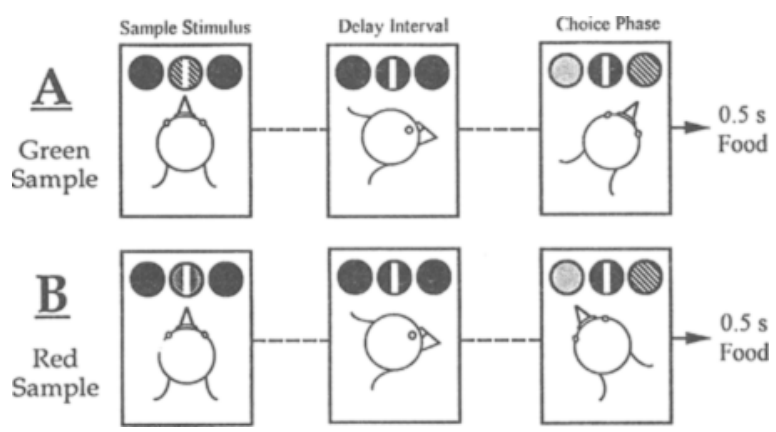

Large-Reward Trials

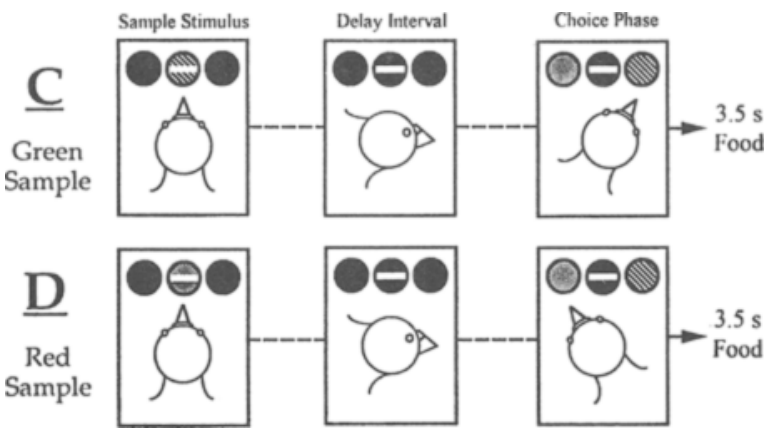

Figure 2. Stimulus arrangements and sequence of events defining the four trial types in Condition 2 of Experiment 1. Trials began with the presentation of the green or red sample stimulus, and the verticalbar or horizontal-bar outcome cue on the center key. Following five responses to this stimulus, the sample extinguished (but the outcome cue remained) and a delay interval of $0.01,1,4$, or 8 sec elapsed before the side keys were lit gneen on the left and red on the right, or vice versa, for the choice phase. On small-reward trials (signaled by the vertical bar), both correct green and correct red responses ( $A$ and $B$ ) produced 0.5 -sec access to wheat. On large-reward trials (signaled by the horizontal bar), both correct responses were followed by 3.5 sec of food access ( $C$ and $D)$. 
was to override any carryover effects from the conditions described in Experiment 2. That is, an SME in Condition 2 required that accuracy on trials presenting the horizontal bar exceed accuracy on trials involving the vertical bar-the opposite to evidence for the DOE in Condition 1 and all conditions of Experiment 2.

Each session in Condition 1 consisted of 128 trials arranged in four blocks of 32 trials. The trials in each block uniquely combined red and green sample stimuli, vertical and horizontal outcome cues, left and right positions of the correct comparison stimulus and four delay intervals. The quasi-random order of trials was different in each block and was constrained so that each stimulus combination occurred equally often and so that red or green, vertical or horizontal, left or right, or any one of the four delay intervals could not appear on more than three consecutive trials. Each session lasted until either 128 trials had been completed or $50 \mathrm{~min}$ had elapsed, whichever came first.

The first 45 sessions of Condition 2 consisted of 128 trials arranged in the same manner as Condition 1 . However, the number of trials per session was reduced to 96 (three blocks of 32 trials) for the final 15 sessions because several birds began to exceed intermittently $85 \%+15 \mathrm{~g}$ of their free-feeding body weights.

The data collected from each session in each condition were the numbers of correct and incorrect responses that a bird made to the left and right side keys on each of the four sample-by-outcome trial types (see Figures 1 and 2). These data were recorded separately for each of the four delay intervals.

\section{Results and Discussion}

Individual-subject analyses of delayed-matching accuracy on DO and SO trials in Condition 1 were based on frequencies of choice responses summed over the last 10 sessions of training (i.e., Sessions 51-60). Consequently, 160 trials (80 presenting the red sample and 80 presenting the green sample) were considered in the calculation of each estimate of accuracy at each delay interval. To approximate the same number of trials contributing to each measure of accuracy, analyses of delayed-matching accuracy on large- and small-reward trials in Condition 2 considered response frequencies summed over the last 13 sessions of training (i.e., Sessions 48-60).

Matching accuracy was initially measured in terms of the proportion of trials on which the bird chose the correct comparison stimulus (PC). Figure 3 shows PC scores for each bird in Condition 1 as a function of the duration of the delay interval on DO and SO trials. The group data were calculated by summing response frequencies across individual birds.

Figure 3 shows that, for all birds, PC on both DO and $\mathrm{SO}$ trials decreased as the delay interval increased. The significance of these trends was supported by the results of two nonparametric monotonic trend tests (Ferguson, 1966). (Using $N=5$ and $k=4$ in each test, $\Sigma S=$ -30 on both DO and SO trials, $p<.05$.) This finding is typical of the form of delay-interval functions in DMTS procedures (Blough, 1959; Roberts, 1972; White, 1985). In addition, Figure 3 illustrates clear evidence of the DOE. That is, for all birds, PC scores were generally higher on trials with different reward durations (DO trials) than they were on trials with the same reward durations (SO trials). Of the 20 possible comparisons,
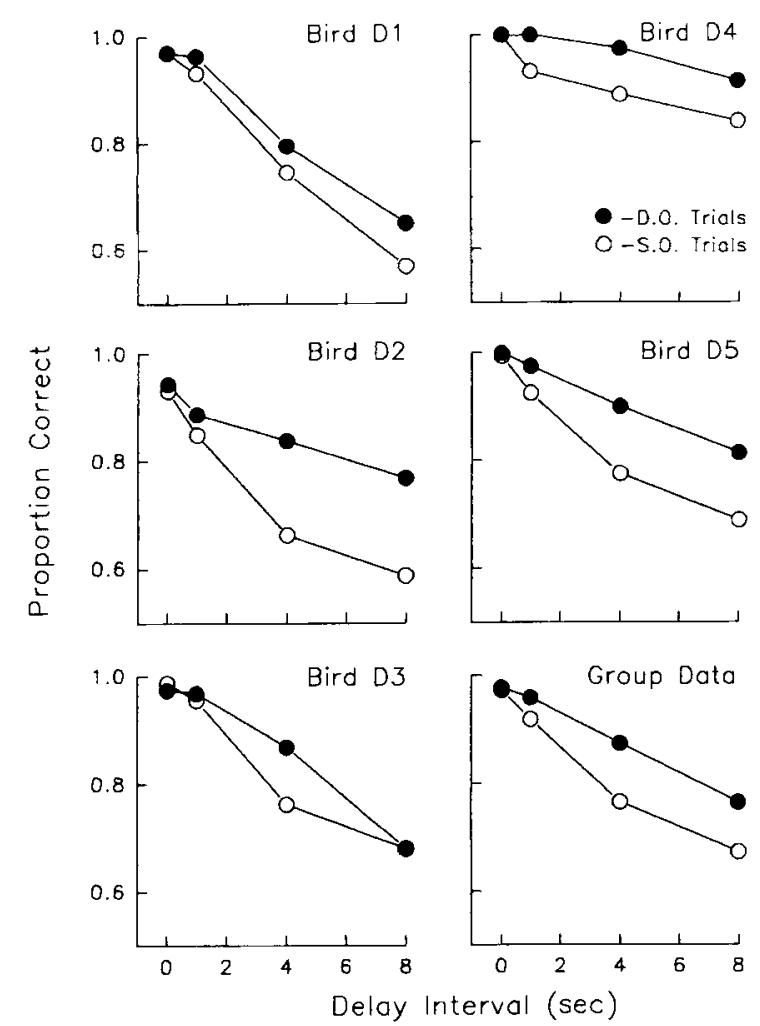

Figure 3. Proportion correct for each bird on different-outcome trials (filled circles) and same-outcome trials (open circles) as a function of the duration of the delay interval in Condition 1 of Experiment 1 .

there were only three instances where accuracy on DO trials was not higher than accuracy on SO trials (Birds $\mathrm{D} 1, \mathrm{D} 3$, and $\mathrm{D} 4$ at $0.01 \mathrm{sec})$. This difference was significant according to a sign test $(p<.05)$. Thus, the birds generally matched more accurately when different rewards were signaled than when the same rewards were signaled even though these outcome cues were irrelevant cues for choice responding.

Superior delay matching on DO trials in the present experiment is consistent with the results of previous studies in which either qualitatively or quantitatively different rewards were arranged to follow correct responses in delayed conditional discriminations (Brodigan \& Peterson, 1976; Honig, Matheson, \& Dodd, 1984; Urcuioli, 1990a, 1990b, 1991). This finding also extends a result reported by Carlson and Wielkiewicz (1976). These authors trained rats in a simultaneous conditional discrimination involving auditory sample stimuli and two response levers. A group presented with a large number of food pellets for one correct response and a small number of pellets for the other correct response learned the discrimination more rapidly than did another group receiving the same amount of food for both responses. The present finding demonstrates that arranging reward magnitudes that are differential with respect 


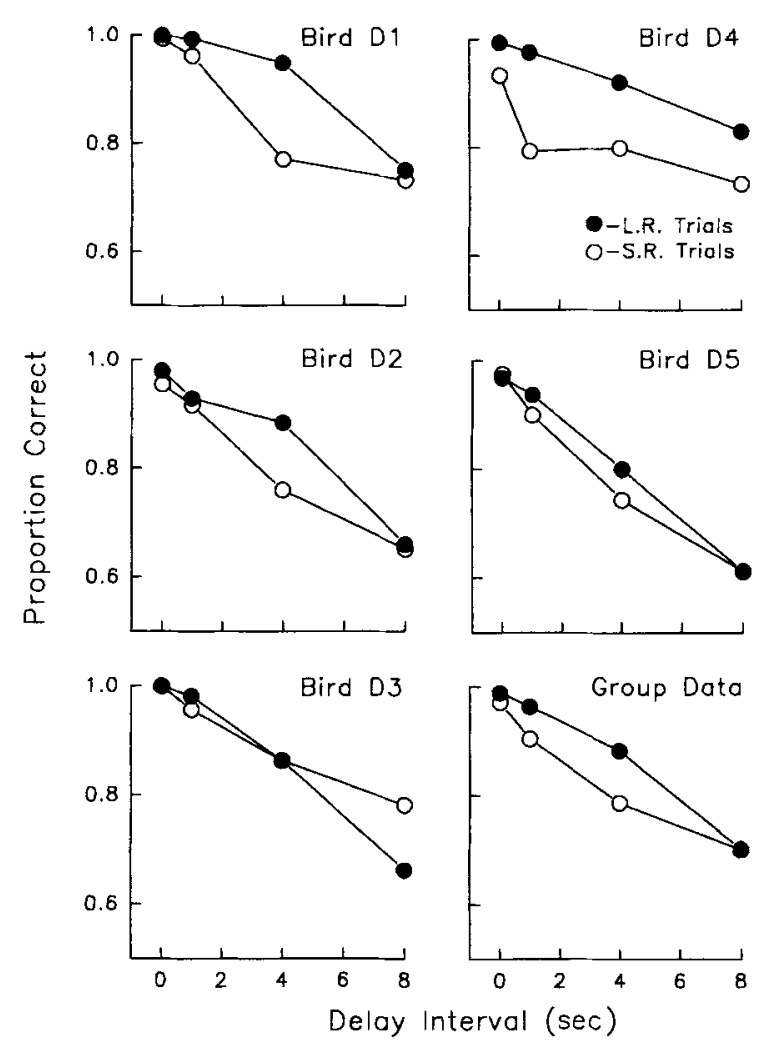

Figure 4. Proportion correct on large-reward trials (filled circles) and small-reward trials (open circles) as a function of the duration of the delay interval in Condition 2 of Experiment 1.

to the sample stimuli also enhances matching accuracy in a DMTS task.

Figure 4 shows PC scores for each bird in Condition 2 as a function of the duration of the delay interval on largeand small-reward trials. Accuracy on large- and smallreward trials again generally decreased as the delay interval increased. The significance of these trends was supported by the results of two nonparametric monotonic trend tests (Ferguson, 1966). (Using $N=5$ and $k=$ 4 in each test, $\Sigma S=-30$ on large-reward trials and $\Sigma S=-28$ on small-reward trials, $p<.05$.) In addition, Figure 4 presents evidence of the SME. That is, PC scores were higher on trials involving longer reward durations (large-reward trials) than they were on trials involving shorter reward durations (small-reward trials). Out of 20 possible comparisons, there were only 3 instances where accuracy on large-reward trials did not exceed accuracy on small-reward trials (Bird D3 at 0.01 and $8 \mathrm{sec}$, and Bird D5 at $0.01 \mathrm{sec}$ ). This difference was significant according to a sign test $(p<.05)$.

An SME in the present experiment replicates the main result of Nevin and Grosch (1990) and extends the range of experimental conditions supporting this phenomenon. In particular, the SME here suggests that procedural differences between the present experiment and Nevin and Grosch's study were not critical for the SME. For exam- ple, in the present experiment, the birds were required to make five "observing" responses to either sample before the trial progressed (i.e., FR 5). Nevin and Grosch, on the other hand, did not require any observing responses to the samples. Instead, each sample stimulus was presented for a fixed time (i.e., FT 5 sec), and pecks at the sample key had no programmed effect. Analyses of the number of responses made to the sample stimuli in their study suggest that this aspect of their procedure may have been critical for the SME. Specifically, Nevin and Grosch reported that all birds pecked both samples more often on large-reward trials than on small-reward trials (see their Figure 5, pp. 302-303). Numerous studies have shown that increasing the number of responses to the sample stimuli improves delayed-matching accuracy (e.g., Cohen, Looney, Brady, \& Aucella, 1976; Roberts, 1972; Sacks, Kamil, \& Mack, 1972; White, 1985). It was therefore possible that Nevin and Grosch observed higher accuracies on large-reward trials because more responses were made to the sample stimuli on these trials. However, by controlling the number of responses to all sample stimuli, the present results suggest that permitting the number of sample-stimulus responses to vary on large- and small-reward trials is not a prerequisite condition for the SME. Rather, differential rates of sample-stimulus responding in Nevin and Grosch's study may have been only collateral behavior quite unrelated to the mechanism underlying the SME.

A further difference between Nevin and Grosch (1990) and the present experiment concerns conditions at the time of reward presentation. Nevin and Grosch illuminated the food magazine with either of two colors depending on whether a long or a short duration of food access was scheduled. (The food magazine was lit blue if the duration was $4.5 \mathrm{sec}$, and yellow if it was $1.5 \mathrm{sec}$.) However, in the present experiment, the food magazine was lit the same color on large- and small-reward trials (i.e., white). An SME in the present experiment therefore suggests that signaling the magnitude of a reward at the time of its delivery was likely to have been an unnecessary aspect of Nevin and Grosch's procedure.

A comparison of the delay-interval functions that characterized the DOE and the SME was conducted by analyzing delayed-matching accuracy in Conditions 1 and 2 in terms of White and McKenzie's (1982) description of DMTS performance. This model adopts a behavioral view of nonhuman short-term memory in which the act of remembering is considered a discriminated operant that is controlled by a temporally distant sample stimulus and maintained by differential reinforcement (see also Harnett et al., 1984). As a result, the accuracy with which subjects "match" in a DMTS task is assumed to reflect, in part, the degree of stimulus control exerted by the samples, or the discriminability between the sample stimuli, at time $t$ since sample presentation. Accordingly, this model measures delayedmatching accuracy in terms of a point estimate of stimulus discriminability $(\log d)$ derived from a model of 
signal-detection performance proposed by Davison and Tustin (1978) and by Nevin (1981). This estimate of stimulus discriminability is the same as $d_{e}$ from Luce's (1963) choice theory and has properties very similar to $d^{\prime}$ (Green \& Swets, 1966). Namely, it is a measure of stimulus discriminability that has no upper bound and is theoretically independent of response bias (see McCarthy \& Davison, 1984, for further explanation). $\log d_{t}(\log d$ at delay-interval $t$ ) is calculated as the logarithm (base 10 ) of the geometric mean of the ratios of correct to incorrect responses on red and green sample trials - that is,

$$
\log d_{t}=0.5 * \log \left(P_{\mathrm{rc}} \cdot P_{\mathrm{gc}} / P_{\mathrm{re}} \cdot P_{\mathrm{ge}}\right),
$$

where $P_{\mathrm{rc}}$ and $P_{\mathrm{re}}$ refer to the number of correct and incorrect responses on red sample trials, respectively, and $P_{\mathrm{gc}}$ and $P_{\mathrm{ge}}$ refer to the number of correct and incorrect responses on green sample trials, respectively.

White and McKenzie (1982; also see White, 1985, 1991) suggested that the discriminability between the samples at time $t\left(\log d_{t}\right)$ is a function of two sets of factors that have independent effects on the form of the delay-interval function. First, time-independent (or attentional) factors affecting the discriminability between the samples when there is no memory requirement determine the initial level of the forgetting function. Second, time-dependent (or memorial) factors affecting the rate at which this discriminability decays with increasing delay intervals determine the slope of the forgetting function. They further suggested that the effect of increasing delay intervals was to degrade sample-stimulus discriminability according to a negative-exponential function of time- that is,

$$
\log d_{t}=\log d_{0} * \exp (-b t),
$$

where $\log d_{t}$ is the discriminability between the sample stimuli at time $t$ since their presentation, $\log d_{0}$ is the discriminability between the stimuli when $t=0$, and $b$ is a constant that describes the rate at which sample discriminability decreases with increasing time $t$. Given the types of manipulations that affect each of these parameters, White $(1985,1991)$ has argued that $\log d_{0}$ measures the initial discriminability between the sample stimuli and $b$ measures the rate at which the samples are forgotten.

Estimates of $\log d_{t}$ at each delay interval on DO and SO trials in Condition 1, and on large- and small-reward trials in Condition 2, were calculated for each bird using Equation 1. Because there were several instances where no errors were made following one or both samples (i.e., $P_{\mathrm{re}}=0$ and $/$ or $P_{\mathrm{ge}}=0$ ), 0.5 was added to all response frequencies irrespective of their values. Snodgrass and Corwin (1988) recommended such a log-linear transformation to permit the calculation of response ratios when any of the four response frequencies was zero. Furthermore, Hautus (1995) reports computer simulations that demonstrate that this transformation modifies uncorrected estimates of $\log d$ in a systematic and predictable manner. Thus, although this transformation will result in estimates of $\log d_{t}$ that are lower than those obtained when the original data are used, the relativity of estimates calculated from transformed data will be preserved.

Equation 2 was then fitted by nonlinear least squares regression to these estimates of $\log d_{t}$ as a function of the duration of the delay interval. This was done using the optimizer in Quattro-Pro for Windows. Table 1 presents the results of this analysis. For each bird, Table 1 shows the estimate of initial discriminability $\left(\log d_{0}\right)$ and the estimate of rate of forgetting $(b)$ that best described the delay-interval functions on DO and SO trials in Condition 1 and on large- and small-reward trials in Condition 2. Also shown is the proportion of variance accounted for in each regression $(V A C)$ along with the mean square error ( $M S E)$ term of each regression.

With only one exception (SO trials for Bird D4), estimates of $\log d_{t}$ as a function of delay-interval duration were described well by Equation 2. VACs ranged from .72 to 1.0 , with a mean of .95 and a median of $.98 . M S E s$ ranged from 0 to .094 , with a mean of .015 and a median of .007. In addition, there were no systematic differences between goodness-of-fit measures across conditions and across trial type within each condition.

Comparisons of the two parameters within and across conditions in Table 1 provide some evidence for quanti-

Table 1

Estimates of the Initial Level of the Delay-Interval Function $\left(\log d_{0}\right)$ and the Slope of the Function (b) That Best Described Each Bird's Performance on Different- and Same-Outcome Trials in Condition 1, and

\begin{tabular}{|c|c|c|c|c|c|c|c|c|c|c|c|c|c|c|c|c|}
\hline \multirow[b]{3}{*}{ Bird } & \multicolumn{8}{|c|}{ Condition 1} & \multicolumn{8}{|c|}{ Condition 2} \\
\hline & \multicolumn{4}{|c|}{ Different-Outcome Trials } & \multicolumn{4}{|c|}{ Same-Outcome Trials } & \multicolumn{4}{|c|}{ Large-Reward Trials } & \multicolumn{4}{|c|}{ Small-Reward Trials } \\
\hline & $\log d_{0}$ & $b$ & $V A C$ & $M S E$ & $\log d_{0}$ & $b$ & $V A C$ & $\overline{M S E}$ & $\log d_{0}$ & $b$ & $V A C$ & $M S E$ & $\log d_{0}$ & $b$ & $V A C$ & $\overline{M S E}$ \\
\hline $\overrightarrow{\mathrm{D} 1}$ & 1.625 & .212 & .88 & .039 & 1.452 & .282 & 1.0 & .000 & 2.259 & .167 & .98 & .008 & 1.867 & .210 & .95 & .016 \\
\hline D2 & 1.111 & .094 & .92 & .004 & 1.077 & .314 & .99 & .001 & 1.787 & .194 & .99 & .004 & 1.327 & .212 & .98 & .004 \\
\hline D3 & 1.722 & .187 & 1.0 & .000 & 1.808 & .294 & .98 & .008 & 2.235 & .252 & 1.0 & .000 & 1.992 & .205 & .89 & .042 \\
\hline D4 & 2.306 & .101 & .98 & .006 & 1.867 & .169 & .72 & .094 & 1.978 & .135 & 1.0 & .000 & 1.312 & .152 & .98 & .003 \\
\hline D5 & 2.019 & .163 & .90 & .033 & 1.854 & .318 & .95 & .022 & 1.469 & .234 & 1.0 & .001 & 1.430 & .286 & .98 & .006 \\
\hline
\end{tabular}
Large- and Small-Reward Trials in Condition 2 of Experiment 1

Note-These parameters were obtained by fitting Equation 2 by nonlinear least squares regression to point estimates of log $d_{t}$ calculated from response frequencies pooled across either the last 10 days of a condition (Condition 1 ) or the last 13 days (Condition 2 ). VAC = the proportion of variance accounted for in each regression, and $M S E=$ the mean square error term for each regression. 
tative differences between the DOE (Condition 1) and the SME (Condition 2). In Condition 1, estimates of $\log d_{0}$ were higher for DO functions than for SO functions for 4 of the 5 birds, and estimates of $b$ were lower for DO functions than for SO functions for all 5 birds. Only the latter of these differences is statistically significant according to a sign test $(p<.05)$. In Condition 2 , estimates of $\log d_{0}$ were higher for large-reward functions than for small-reward functions for all 5 birds, and estimates of $b$ were lower for large-reward functions than for smallreward functions for 4 of the 5 birds. Only the former of these differences is statistically significant according to a sign test $(p<.05)$. Thus, these differences suggest that signaling different rewards at least reliably attenuates the rate at which sample discriminability declines with increasing delay intervals relative to signaling the same rewards, whereas signaling larger rewards for both correct responses at least reliably increases the initial level of the delay-interval function. It is not so clear whether signaling different rewards increases the initial level of the delay-interval function or whether signaling larger rewards decreases the slopes of these functions.

The individual-subject data provided in Nevin and Grosch (1990) were reanalyzed to investigate further whether signaling larger rewards might affect only the initial level of the delay-interval function. The PC scores appearing in their Figure 1 were converted to estimates of logit $p_{t}$ by a logistic transformation. In the absence of a bias to choose one comparison stimulus more often than the other, $\operatorname{logit} p_{t}$ (or $\log [\mathrm{PC} /(1-\mathrm{PC})]$ at delay interval $t$ ) is equivalent to $\log d_{t}$. Assuming no systematic biases in Nevin and Grosch's data seems reasonable on the grounds that they scheduled equal reinforcer durations for the two correct responses. Table 2 shows the estimates of initial discriminability (logit $p_{0}$ ) and rate of forgetting $(b)$ that best described the large- and smallreward delay-interval functions for each bird in Nevin and Grosch's study. Also shown are the VAC and the MSE of each regression.
Table 2 shows that estimates of logit $p$ as a function of delay-interval duration were generally described well by Equation 2. VACs ranged from .57 to 1.0, with a mean of .85 and a median of .89 . MSEs ranged from 0 to .029 , with a mean of .011 and a median of .009 . In addition, there were no systematic differences between goodnessof-fit measures for large- and small-reward trials. A comparison of logit $p_{0}$ and $b$ estimates for the two trial types confirms the reliability, and lack thereof, of the effects found in the present experiment. The delay-interval functions describing matching accuracies on large- and small-reward trials consistently differed in their initial levels (estimates of logit $p_{0}$ ), but did not differ in their slopes (estimates of $b$ ). More specifically, estimates of $\operatorname{logit} p_{0}$ were higher on large-reward trials than on smallreward trials for all 5 birds, whereas estimates of $b$ were lower on large-reward trials than on small-reward trials for only 2 of the 5 birds. Taken together with the results of Condition 2, these results suggest that signaling larger rewards for both correct responses improves delayedmatching accuracies only by enhancing the initial discriminability between the samples and not by reducing the rate at which sample discriminability decreases with increasing delay intervals.

Figure 5 summarizes the quantitative differences between the DOE and the SME that were observed in the present experiment (Table 1). The top panel of Figure 5 shows mean estimates of $\log d_{t}$ on DO and SO trials plotted as a function of delay-interval duration. The bottom panel shows mean estimates of $\log d_{t}$ on large- and small-reward trials also plotted as a function of delayinterval duration. These estimates of $\log d_{t}$ were calculated by pooling the raw response frequencies across subjects. No log-linear transformation was performed on these data. The solid lines drawn through the data represent the negative-exponential function (Equation 2) that best described each set of data. In addition, the estimates of $\log d_{0}$ and $b$ that characterized these functions, along with VACs, are shown in each panel. The dif-

Table 2

The Results of a Reanalysis of Delayed-Matching Accuracies on Large- and Small-Reward Trials in the Study by Nevin and Grosch (1990)

\begin{tabular}{rccccccccc}
\hline & \multicolumn{4}{c}{ Large-Reward Trials } & & \multicolumn{3}{c}{ Small-Reward Trials } \\
\cline { 2 - 5 } \cline { 6 - 9 } Bird & logit $p_{0}$ & $b$ & $V A C$ & $M S E$ & & $\operatorname{logit} p_{0}$ & $b$ & VAC & $M S E$ \\
\hline 3 & 2.113 & .029 & .84 & .026 & & 0.997 & .028 & 1.0 & .000 \\
8 & 1.623 & .098 & .93 & .015 & & 1.137 & .090 & .91 & .009 \\
11 & 1.441 & .010 & .57 & .008 & & 1.047 & .006 & .58 & .002 \\
48 & 0.925 & .092 & .96 & .003 & & 0.489 & .432 & .99 & .000 \\
49 & 1.591 & .127 & .87 & .029 & & 1.023 & .150 & .82 & .019 \\
\hline
\end{tabular}

Note-The negative-exponential function given by Equation 2 was fitted by nonlinear least squares regression to logit $p_{t}$ measures derived from data presented in Nevin and Grosch's (1990) Figure 1. Logit $p_{t}$ is given by $\log [P C /(1-P C)]$ at delay interval $t$ where $P C$ refers to the proportion of trials on which the correct comparison stimulus was chosen. The parameter values that best described the initial level of the delayinterval functions (logit $p_{0}$ ) and the slope of these functions $(b)$ are shown together with the proportion of variance accounted for in each regression $(V A C)$ and the mean square error (MSE) for each regression. 

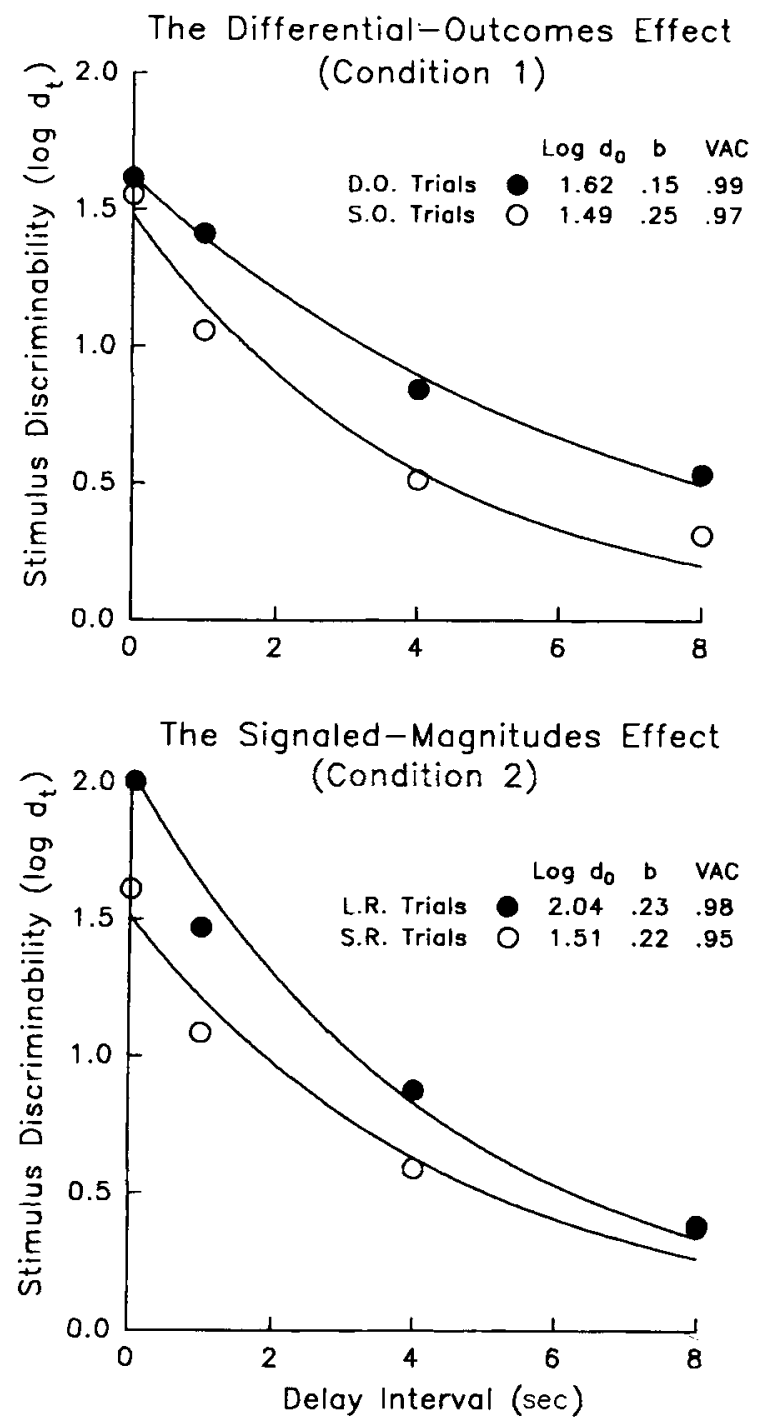

Figure 5. Estimates of $\log d$, on different-outcome, same-outcome, large-, and small-reward trials calculated from group data in Conditions 1 and 2 of Experiment 1 and plotted as a function of the duration of the delay interval on these trials. The solid lines depict the negative-exponential function (Equation 2) that best described each set of data. The estimates of $\log d_{0}$ and $b$ that characterized each fitted function, along with the proportion of variance accounted for in each regression (VAC), are shown above the data in each panel.

ferences between estimates of $\log d_{0}$ and estimates of $b$ on DO and SO trials and on large- and small- reward trials are similar to those seen in analyses of individual birds (Table 1). Signaling differential rewards in Condition 1 increased the initial level of the delay-interval function $\left(\log d_{0}\right)$ and decreased the slope of this function (b), whereas signaling larger rewards in Condition 2 only increased initial level and did not affect slope. Furthermore, the slopes of both large- and small-reward functions $(0.23$ and 0.22 , respectively) were very simi- lar to the slope of the SO function (0.25), and the initial level of the SO function (1.49) was similar to the initial level of the small-reward function (1.51).

Evidence for quantitative differences between the DOE and the SME is consistent with recent theoretical accounts of each phenomena. Accounts of the SME have often appealed to time-independent or "attentional" processes (see Nelson, 1976), whereas accounts of the DOE have often described time-dependent or "memorial" processes (see Honig \& Thompson, 1982). For example, Nevin and Grosch (1990) argued that a difference between only the initial level of the delay-interval functions on large- and small-reward trials is consistent with an attentional interpretation of the SME where the effect of signaling larger rewards is to enhance a subject's attention to the samples on large-reward trials and thus enhance the discriminability between the samples on these trials. In contrast, different rates of forgetting on DO and SO trials imply that factors that attenuate the effect of increasing delay intervals operate in the DOE.

Outcome-expectancy theory (Peterson \& Trapold, 1982; Trapold, 1970) is one explanation that claims different remembering strategies on DO and SO trials. According to this theory, the facilitation of matching accuracies by arranging DOs reflects the control of choice responding by differential expectancies of these rewards. That is, given a sufficient number of pairings of each of the sample stimuli with their respective rewards, the presentation of a sample elicits (by Pavlovian processes) an expectancy of the reinforcer that mediates the delay interval and subsequently exerts stimulus control over choice responding. This discriminative control by differential expectancies apparently develops because each correct response has been differentially reinforced in the presence of a specific reward expectancy. Thus, accuracies in a procedure facilitating differential reinforcer expectancies exceed those in a SO procedure by providing subjects with an additional cue for correct responding (Honig et al., 1984; Peterson, 1984; Peterson \& Trapold, 1982; Trapold, 1970; Urcuioli, 1990a). Moreover, to the extent that delayed-matching accuracies in a DO procedure decline slower with increasing delay intervals, reinforcer expectancies are presumed to be less susceptible to decay than are memories of the sample stimuli (DeLong \& Wasserman, 1981; Peterson et al., 1987).

It is important to note that considerable debate has centered around the nature of such outcome expectancies. Several authors have argued that these expectancies exist within the organism's central nervous system and are represented as neural activity (e.g., Trapold, 1970; Trapold \& Overmier, 1972). Others have argued that differential expectancies may be sample-specific overt chains of behavior during the delay interval, where the proprioceptive or interoceptive stimuli arising from these behaviors are presumed to provide either additional cues or more salient cues that replace control by the sample stimuli (Brodigan \& Peterson, 1976; Peterson \& Trapold, 1980). However, empirical investiga- 
tions of the function of observed sample-specific behavior have provided mixed results (see DeLong \& Wasserman, 1981). These results suggest that although the stimulus properties of sample-specific responding cannot alone account for the DOE, differential behavior after each sample might attenuate the effect of increasing delay intervals on matching accuracy by persisting after the offset of the samples and being more salient cues than the sample stimuli at the time of the choice phase. Furthermore, Peterson (1984) suggested that whether reward expectancies are represented centrally or as observable behavior is a moot point and is not critical for outcome-expectancy theory.

The present results also have implications for an alternative account of the mechanism underlying the DOE. In this account, associating DOs with the two sample stimuli adds another stimulus dimension to the discrimination and enhances delayed-matching accuracy by enhancing the discriminability between the samples (see Edwards, Jagielo, Zentall, \& Hogan, 1982; Honig et al., 1984; Peterson \& Trapold, 1982). Accordingly, researchers advocating this account often liken the DOE to the "acquired distinctiveness of cues" phenomenon (Lawrence, 1949; see also Edwards et al., 1982; Peterson \& Trapold, 1982). However, the present results suggest that the DOE is quite distinct from the acquired distinctiveness of cues phenomenon. This account does not predict an effect of signaling DOs on rates of forgetting. Moreover, the present results, in conjunction with previous quantitative analyses of DMTS performance, suggest that DOs do not reduce rates of forgetting by enhancing the discriminability between the stimuli. Thus, although signaling DOs may have had both of these effects, it is quite likely that they were not causally related and in fact represented independent effects. For example, a description of the SME in terms of Equation 2 (Table 2) revealed that signaling larger outcomes enhanced the discriminability between the stimuli without reducing rates of forgetting. In addition, White and his colleagues have fitted Equation 2 to numerous manipulations of the DMTS procedure (see White, 1991), and parameter invariance was observed. That is, in all cases, variation of some aspect of the procedure affected either the initial discriminability between the samples $\left(\log d_{0}\right)$ or rates of forgetting $(b)$, but not both.

Quantitative differences between the DOE and the SME in the present experiment suggest strongly that these two phenomenon are independent effects of signaling the rewards in a DMTS task. In particular, by involving an effect on rates of forgetting, the DOE is likely to involve mechanisms distinct from those operating in the SME. Indeed, in many prior studies of the DOE, control groups have received both rewards offered to the experimental group but uncorrelated with the sample stimuli and the two correct responses (Carlson \& Wielkiewicz, 1976; Overmier, Bull, \& Trapold, 1971; Peterson et al., 1978; Peterson et al., 1980). A DOE under these conditions implies that the mechanism(s) underlying the SME cannot alone account for the DOE.
However, it is not entirely clear whether the mechanism underlying the SME in Condition 2 at least contributed to the DOE observed in Condition 1. Although only 4 of the 5 birds revealed higher estimates of $\log d_{0}$ on DO trials than on SO trials, we cannot rule out the possibility that signaling different rewards does enhance the initial discriminability between samples. If this were true, then a common effect on initial discriminability of signaling different rewards and of signaling larger rewards may well imply that the mechanism producing the SME in the present procedure also contributed to the DOE. For instance, if we accept the explanation offered by Nevin and Grosch (1990) for the SME, then an enhanced discriminability between samples on DO trials (for 4 of the birds) may also have resulted from greater attention to the samples on these trials. This possibility is favored by a difference between the overall reinforcer magnitudes provided on DO trials and those provided on SO trials (in Condition 1). In this condition, the mean duration of food access on DO trials was $2 \mathrm{sec}$ (i.e., [3.5 sec $+0.5 \mathrm{sec}] / 2$ ), whereas on SO trials it was only $1.5 \mathrm{sec}$. Thus, because the average value of the reward obtained on DO trials exceeded the value of the rewards obtained on SO trials, the initial discriminability between the samples on DO trials may have been enhanced by the anticipation of a larger average reward on these trials. Experiment 2 investigated this possibility and provided further data with which to assess the reliability of differences between $\log d_{0}$ on DO and SO trials.

\section{EXPERIMENT 2}

Experiment 2 examined further the independence of the DOE and the SME observed in Experiment 1. It assessed whether overall higher delayed-matching accuracies on DO trials than on SO trials in Experiment 1 were related to the mean duration of food access on DO trials exceeding that on SO trials. Specifically, we arranged further conditions to assess the reliability of differences between $\log d_{0}$ on DO and SO trials and asked whether birds performed more accurately on DO trials involving the shorter retention intervals because the DO cue signaled an overall larger reward than was signaled by the SO cue.

Condition 2 of Experiment 1 indicated that signaling a larger reward increased the initial level of the delayinterval function. This result is consistent with an attentional interpretation of the SME (Nevin \& Grosch, 1990) where signals for larger rewards enhance a subject's attention to the samples and, in so doing, enhance the initial discriminability between the samples. If the birds that showed higher estimates of $\log d_{0}$ on DO trials than on SO trials in Condition 1 of Experiment 1 discriminated a difference between the overall reinforcer magnitude on DO trials and that on SO trials, and overall reward value modulated attention to the samples, then the difference between the initial levels of these delay-interval functions may represent a SME component to the DOE we measured. 
In Experiment 2, the same pigeons used in Experiment 1 were trained in a DMTS task similar to that arranged in Condition 1 of Experiment 1 . A vertical bar superimposed on the sample stimuli signaled different durations of food access for the two correct responses (DO trials), and a horizontal bar signaled equal durations of food access (SO trials). The duration of food access for correct responses on DO trials remained constant across conditions. Correct responses on greensample DO trials always produced 3.5 -sec access to food, and correct responses on red-sample trials always produced $0.5-\mathrm{sec}$ access to food. However, the duration of food access on SO trials was varied across conditions $(0.5,3.5$, or $1.5 \mathrm{sec}$ of food access for both correct responses). If the initial discriminability component of the DOE in Experiment 1 reflected a preference for the rewards on DO trials, then scheduling 0.5 -sec food access on SO trials should increase the initial discriminability difference on the two trial types relative to scheduling $1.5 \mathrm{sec}$ on SO trials. Similarly, scheduling food-access times on SO trials $(3.5 \mathrm{sec})$ that exceed the mean access time on DO trials $(2 \mathrm{sec}$ ) should either attenuate the initial discriminability difference relative to the 0.5 - and 1.5 -sec conditions or eliminate the difference altogether.

\section{Method}

The subjects, apparatus, and stimuli used here were identical to those used in Experiment 1.

The sequence of stimulus events defining the four trial types was identical to that in Condition 1 of Experiment 1 (see Figure 1). The birds were required to match red and green samples by choosing between red and green comparison stimuli after delay intervals of $0.01,1,4$, and $8 \mathrm{sec}$. However, unlike Experiment 1, the duration of food access for correct responses on SO trials varied across conditions. In all conditions, the vertical bar signaled different durations of food access (DOs) for the two correct responses, and the horizontal bar signaled equal durations of food access (SOs). The durations of food access on DO trials were held constant across conditions ( $3.5 \mathrm{sec}$ for correct green and $0.5 \mathrm{sec}$ for correct red). In Condition 1, correct responses on SO trials produced 0.5 -sec food access. In Condition 2, correct responses on SO trials produced $3.5-\mathrm{sec}$ food access. In Condition 3 , they produced $1.5 \mathrm{sec}$

Each session lasted until either 128 trials had been completed or $50 \mathrm{~min}$ had elapsed, whichever came first. A quasi-random sequence determined the order in which the trials were presented (see Experiment 1 for further details). All birds received a minimum of 30 sessions of training in each condition.

The data collected from each session were the numbers of correct and incorrect responses that a bird made to the left and right side keys and the times taken to complete the FR 5 requirement to the sample on each of the four sample-by-outcome trial types (i.e., red-sample DO trials, green-sample DO trials, red-sample SO trials, and green-sample SO trials). The number and type of correct and incorrect responses made were recorded separately for each of the four delay intervals.

\section{Results and Discussion}

Analyses of delayed-matching accuracies on DO and SO trials in Conditions 1-3 were based on choiceresponse frequencies summed over the last 10 sessions of training in each condition (Sessions 21-30). For each bird, Figure 6 shows the proportion of correct responses (PC) made on DO and SO trials at each delay interval in Conditions 1-3. The duration of food access arranged on SO trials in each condition is shown above each panel.

Figure 6 shows that PC on both trial types in all conditions generally decreased as the delay interval increased. The significance of these trends was confirmed by six nonparametric tests for monotonic trends (Ferguson, 1966) conducted on PC scores on DO and SO trials in each of the three conditions. (Using $N=5$ and $k=4$ in each test, $\Sigma S=-26,-30,-30,-30,-28$, and -30 on DO and SO trials in Conditions 1,2 , and 3, respectively, $p<.05$.) In addition, the DOE was evident for all birds in all conditions. This can be seen in PC scores on DO trials being generally higher than PC scores on SO trials. A sign test conducted on individual-subject data in each condition confirmed the significance of this difference $(p<.05)$. Of particular importance is that each bird showed higher matching accuracies on DO trials

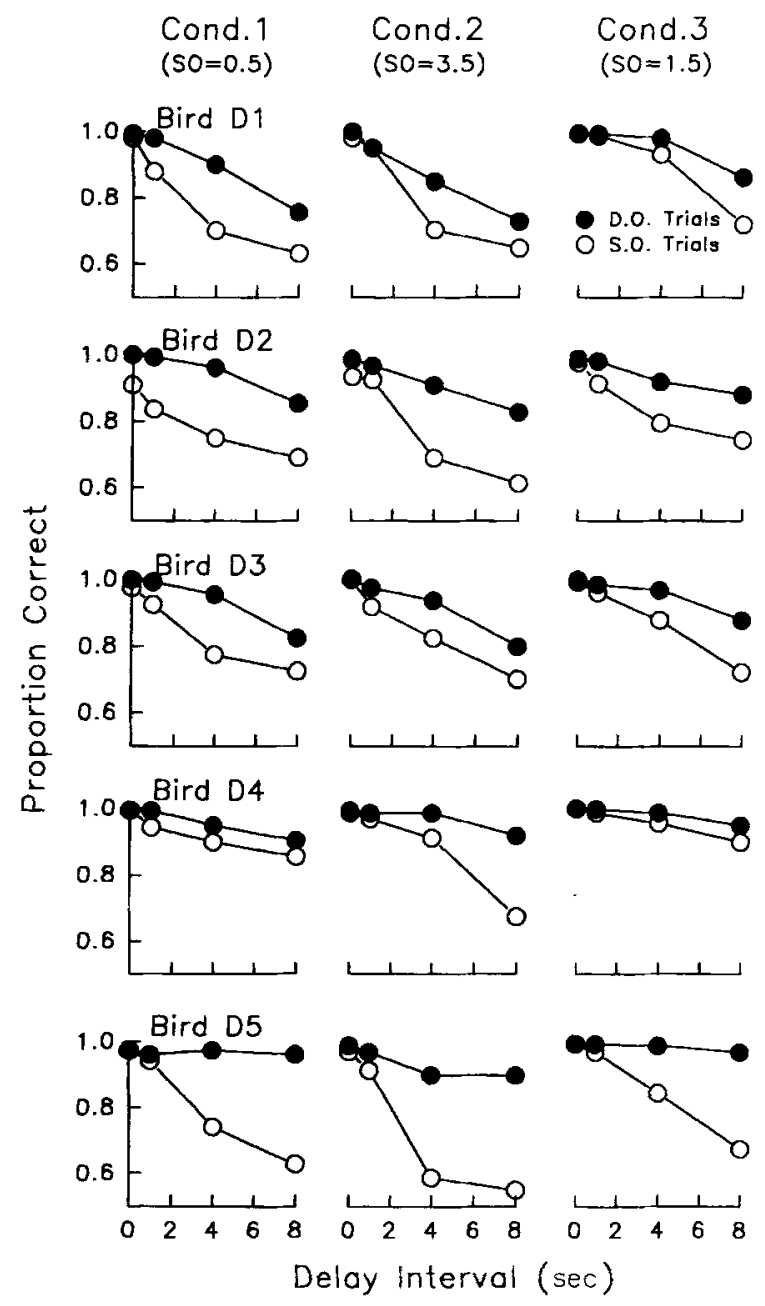

Figure 6. Proportion correct for each bird on different-outcome trials (filled circles) and same-outcome trials (open circles) in Conditions 1, 2, and 3 of Experiment 2 plotted as a function of the duration of the delay interval. 
than on SO trials when the duration of food access on SO trials exceeded the mean arranged on DO trials (i.e., Condition 2 where 3.5-sec food access was arranged for SO trials).

Estimates of $\log d_{t}$ (Equation 1) for each bird on DO and SO trials in each condition were calculated from choice-response frequencies following a log-linear transformation of the raw data (see Experiment 1). The negative-exponential function described by Equation 2 was then fitted to $\log d_{t}$ estimates as a function of delayinterval duration in the same manner as described in Experiment 1 . For each bird, Table 3 shows the estimates of $\log d_{0}$ and $b$ that best described the delay-interval functions on DO and SO trials in Conditions 1, 2, and 3, and $V A C$ s and corresponding MSEs.

The values of $V A C$ and $M S E$ in Table 3 reveal that Equation 2 described each set of data reasonably precisely. (VACs ranged from .15 to 1.0 , with a mean of .92 and a median .95. MSEs ranged from .001 to .061 , with a mean of .011 and a median of .007.) For each bird in each condition, estimates of $\log d_{0}$ were higher and estimates of $b$ were lower on DO trials than on SO trials. Taken together with the results of Experiment 1, these differences confirm that the DOE involves both timeindependent factors affecting the initial level of the delay-interval function and time-dependent factors affecting the slope of this function. Condition 1 of Experiment 1 and all three conditions of Experiment 2 provide 20 comparisons with which to assess differences be- tween $\log d_{0}$ and $b$ on DO and SO trials. Of these comparisons, there were 19 cases where $\log d_{0}$ was higher on DO trials than on SO trials and 20 cases where $b$ was lower on DO trials than on SO trials. Both of these differences were significant according to a sign test $(p<$ .05 ). Furthermore, when a similar analysis was conducted using $d^{\prime}$ (Green \& Swets, 1966) as the dependent measure in Equation 2, estimates of $d_{0}^{\prime}$ and $b$ differed from those obtained when Equation 2 proper was used, but precisely the same ordinal relations were observed.

All birds in the present study received Condition 2 of Experiment 1 immediately after Condition 3 of Experiment 2 . This meant that Conditions 1 and 2 of Experiment 1 were separated by considerable training. Ideally, the order of conditions in Experiment 1 would be counterbalanced, and this feature of the design might suggest that some caution is warranted when interpreting differences between the delay-interval functions obtained in the two conditions of Experiment 1. However, a comparison of the parameter values shown in Table 3 with those shown in Table 1 suggests that quantitative characteristics of the SME (Condition 2 of Experiment 1) were unlikely to have been influenced by order effects. In Condition 3, DO trials were signaled by a vertical bar over either sample and SO trials were signaled by a horizontal bar. In Condition 2 of Experiment 1 (which followed), the outcome cue that was associated with DOs in Condition 3 now signaled smaller rewards and the outcome cue that was associated with SOs now signaled

Table 3

Estimates of the Initial Level of the Forgetting Function $\left(\log d_{0}\right)$ and the Slope of the Function (b) That Best Described Estimates of Sample Discriminability at Each Delay Interval $\left(\log d_{f}\right)$ on Different- and Same-Outcome Trials for Each Bird in Each Condition of Experiment 2

\begin{tabular}{|c|c|c|c|c|c|c|c|c|}
\hline \multirow[b]{2}{*}{ Bird } & \multicolumn{4}{|c|}{ Different-Outcome Trials } & \multicolumn{4}{|c|}{ Same-Outcome Trials } \\
\hline & $\log d_{0}$ & $b$ & $V A C$ & $M S E$ & $\log d_{0}$ & $b$ & $V A C$ & $M S E$ \\
\hline \multicolumn{9}{|c|}{ Condition $1(\mathrm{SO}=0.5 \mathrm{sec})$} \\
\hline D1 & 2.021 & .174 & .99 & .002 & 1.730 & .358 & .98 & .008 \\
\hline D2 & 2.216 & .121 & 1.0 & .001 & 1.052 & .149 & .92 & .007 \\
\hline D3 & 2.163 & .072 & .99 & .001 & 1.641 & .215 & .92 & .020 \\
\hline D4 & 1.987 & .046 & .94 & .004 & 1.839 & .106 & .91 & .015 \\
\hline D5 & 1.688 & .003 & .15 & .002 & 1.521 & .248 & .99 & .002 \\
\hline \multicolumn{9}{|c|}{ Condition $2(\mathrm{SO}=3.5 \mathrm{sec})$} \\
\hline D1 & 2.043 & .247 & .94 & .027 & 1.639 & .301 & .98 & .008 \\
\hline D2 & 1.738 & .117 & .96 & .008 & 1.208 & .239 & .94 & .010 \\
\hline D3 & 2.060 & .160 & .94 & .019 & 1.997 & .320 & .87 & .060 \\
\hline D4 & 2.034 & .066 & .79 & .030 & 1.748 & .165 & .98 & .004 \\
\hline D5 & 1.724 & .103 & .84 & .025 & 1.461 & .425 & .98 & .004 \\
\hline \multicolumn{9}{|c|}{ Condition $3(\mathrm{SO}=1.5 \mathrm{sec})$} \\
\hline D1 & 2.092 & .098 & .91 & .022 & 2.011 & .170 & .99 & .004 \\
\hline D2 & 1.720 & .097 & .95 & .007 & 1.493 & .190 & .95 & .009 \\
\hline D3 & 2.142 & .100 & .98 & .003 & 1.856 & .205 & .97 & .009 \\
\hline D4 & 2.281 & .060 & .97 & .004 & 2.141 & .111 & .98 & .004 \\
\hline D5 & 2.019 & .034 & .91 & .004 & 1.913 & .242 & .99 & .003 \\
\hline
\end{tabular}

Note-These estimates were obtained by fitting Equation 2 by nonlinear least squares regression to point estimates of $\log d_{t}$ calculated from response frequencies pooled across the last 10 days of a condition. $V A C=$ the proportion of variance accounted for in each regression, and $M S E=$ the mean square error term for each regression. 
larger rewards. Therefore, an SME in Condition 2 required that accuracy on trials presenting the horizontal bar now exceed accuracy on trials involving the vertical bar-the opposite to evidence for the DOE in Condition 3 . A comparison of the lower portion of Table 3 with the lower portion of Table 1 shows evidence of just such a reversal of accuracy with respect to the outcome cues. For all 5 birds, estimates of $\log d_{0}$ decreased from DO trials in Condition 3 to small-reward trials in Condition 2, and estimates of $b$ increased from DO trials in Condition 3 to small-reward trials in Condition 2. Both of these differences were significant according to a sign test $(p<.05)$. Furthermore, in a reanalysis of data reported by Berryman, Cumming, and Nevin (1963), White (1985) found that estimates of $\log d_{0}$ tend to improve with continued training in standard (SO) DMTS procedures, but estimates of $b$ do not change over the course of acquisition. These trends are clearly different from those seen in the present data. Thus, it seems unlikely that the differences between the DOE and the SME in the present experiment reflect the order of treatments rather than the nature of these treatments.

To examine whether the difference between the overall magnitudes of reward on DO and SO trials affected the difference between the initial levels and slopes of DO and SO delay-interval functions, the estimates of log $d_{0}$ and $b$ shown in Table 3 are plotted as a function of the food-access durations on SO trials in Figure 7. The left panel presents estimates of $\log d_{0}$ for each bird on DO and SO trials, and the right panel presents estimates of $b$.

The analysis presented in Figure 7 suggests that the duration of food access on SO trials, and therefore the difference between overall reward magnitudes on DO and SO trials, did not affect estimates of $\log d_{0}$ on either DO or SO trials within each condition in any systematic way. The absence of trends in both estimates on DO and SO trials was supported by the results of four nonparametric monotonic trend tests (Ferguson, 1966). (Using $N=5$ and $k=3$ in each test, $\Sigma S=-1$ for estimates of $\log d_{0}$ on both DO and SO trials, and $\Sigma S=9$ and $\Sigma S=$ 7 for estimates of $b$ on DO and SO trials, respectively, $p<.05$.) More importantly, Figure 7 shows that the difference between estimates of $\log d_{0}$ on DO and SO trials did not systematically decrease as the duration of food access on SO trials increased. This was also supported by a nonparametric trend test (Ferguson, 1966) conducted on the difference between $\log d_{0}$ on DO and SO trials. (Using $N=5$ and $k=3, \Sigma S=-1, p<.05$.) In addition, the difference between estimates of $b$ on DO and SO trials did not significantly increase or decrease as a function of the food access provided on SO trials. (Using $N=5$ and $k=3, \Sigma S=-3, p<.05$.)

The failure to find systematic effects of SO trial foodaccess durations on estimates of initial discriminability $\left(\log d_{0}\right)$ on DO and SO trials suggests that an SME is unlikely to have underlain the higher initial levels of DO delay-interval functions than SO functions in this procedure. In other words, it is unlikely that the $\log d_{0}$ component of the DOE resulted from arranging an overall
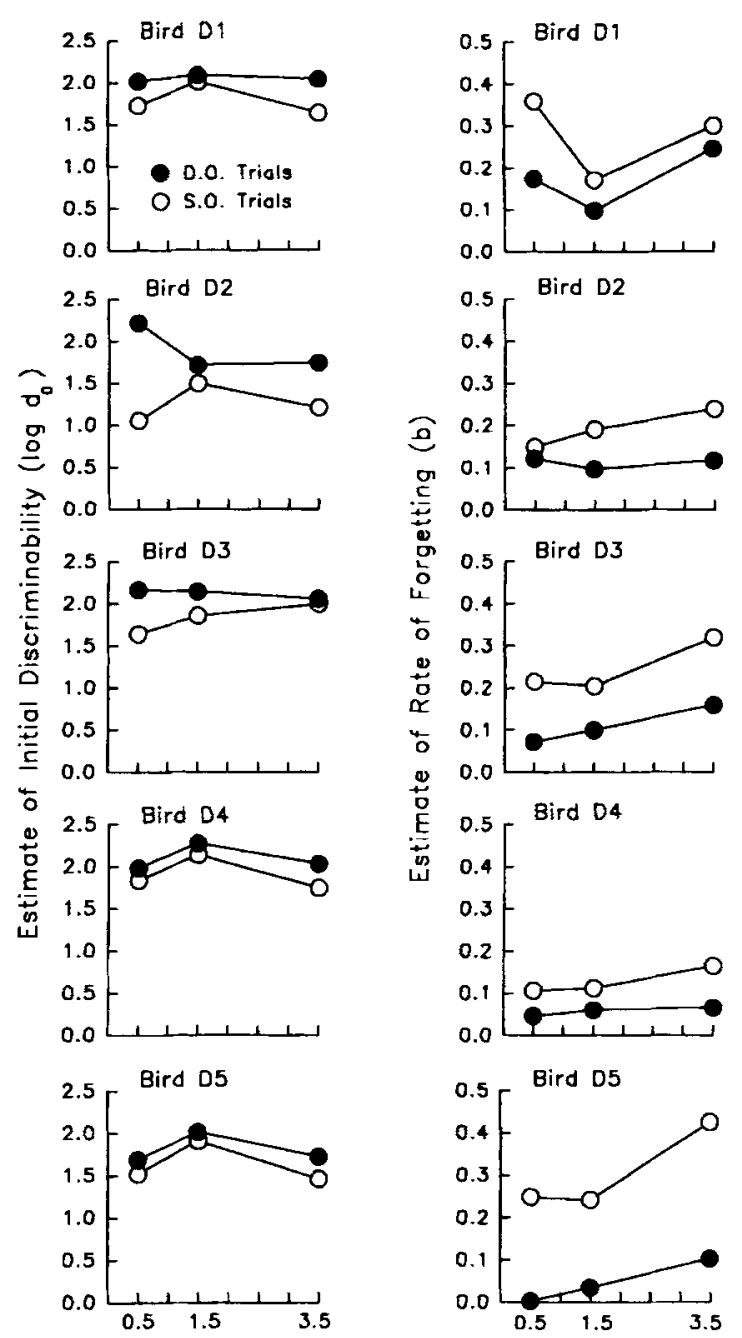

Duration of Food Access on Same-Outcome Trials (sec)

Figure 7. Estimates of the initial level of the delay-interval function (log $d_{0}$, left panels) and the slope of these functions ( $b$, right panels) for each bird on different-outcome trials (filled circles) and sameoutcome trials (open circles) plotted as a function of the duration of food access provided for correct responses on same-outcome trials in Conditions 1, 2, and 3 of Experiment 2. These data are shown in Table 3.

larger reward magnitude on DO trials than on SO trials. In addition, the failure to find systematic effects of SO trial food-access durations on estimates of rates of forgetting $(b)$ on DO and SO trials is consistent with a main finding in Experiment 1-namely, that signaling an overall larger reward for both correct responses does not affect the slope of the delay-interval function. Instead, the present results suggest that the anticipation of differential rewards on DO trials is sufficient to improve delayed-matching accuracies relative to an SO control. Moreover, these results suggest that the effect of DOs can in fact overshadow any facilitative effect of larger rewards on SO trials.

The viability of a signaled-magnitude explanation of the initial discriminability component of the DOE is also 
questionable when one considers this argument in more detail. In the SME demonstrated in Experiment 1 and by Nevin and Grosch (1990), higher accuracy on trials involving the larger of two rewards was necessarily controlled by the outcome cues signaling these rewards. By being associated with differential rewards, these outcome cues permitted a subject to anticipate the preferred reward. The argument that a similar mechanism might be responsible for the DOE requires an important assumption-namely, one must assume that although the magnitude of only one of the DO trial rewards exceeded those provided on SO trials (i.e., green-sample DO trials), the mean magnitude of reward on DO trials was anticipated upon the presentation of the DO cue. This assumption requires that the subjects were treating the outcome cues and the sample stimuli as independent stimuli. However, it is quite plausible that each outcomecue-sample-stimulus combination in this paradigm functioned as a compound stimulus signaling the specific duration of food access on that trial. If the subjects had learned to anticipate the specific reward magnitudes on DO trials, then it is unlikely that the DO cue controlled greater attention to both samples in Condition 1 of Experiment 1 and Conditions 1 and 3 of Experiment 2, because only one of the DO trials provided a larger reward than the SO trials in these conditions. Clearly, without such a preference, higher initial discriminabilities on DO trials cannot be explained by appealing to the SME.

Findings reported by Nevin and Grosch (1990) suggest that an analysis of the rates of responding to each sample-outcome-cue compound might aid in examining the stimulus control of reward expectancies in the present study. As mentioned earlier, Nevin and Grosch reported that birds pecked both sample stimuli more often on large-reward trials than on small-reward trials even though each sample was presented for a fixed time and independent of responding. As well, several studies have found that response rates in free-operant situations are higher in the presence of a stimulus correlated with more preferred rewards than in the presence of a stimulus correlated with smaller rewards (e.g., Nevin, Mandell, \& Yarensky, 1981; Shettleworth \& Nevin, 1965). These findings suggest that differential responding on largeand small-reward trials in Nevin and Grosch's study reflected an expectancy of the magnitude of the reward on each trial and that the expectancy of a larger reward accelerated rates of responding to the samples.

In the present experiment, the time taken for a bird to complete the response requirement to the sampleoutcome-cue stimulus (FR 5) was recorded for each of the four sample-by-outcome trial types. Figure 8 shows the rates at which the birds responded to the sample stimulus on each of the four sample-outcome-cue compounds (i.e., the vertical bar over the red sample, $\mathrm{R}-\mathrm{V}$; the vertical bar over the green sample, G-V; the horizontal bar over the red sample, $\mathrm{R}-\mathrm{H}$; and the horizontal bar over the green sample, G-H) in each condition of Experiment 2. These response rates are expressed as the
Cond.1 Cond.2 Cond. 3
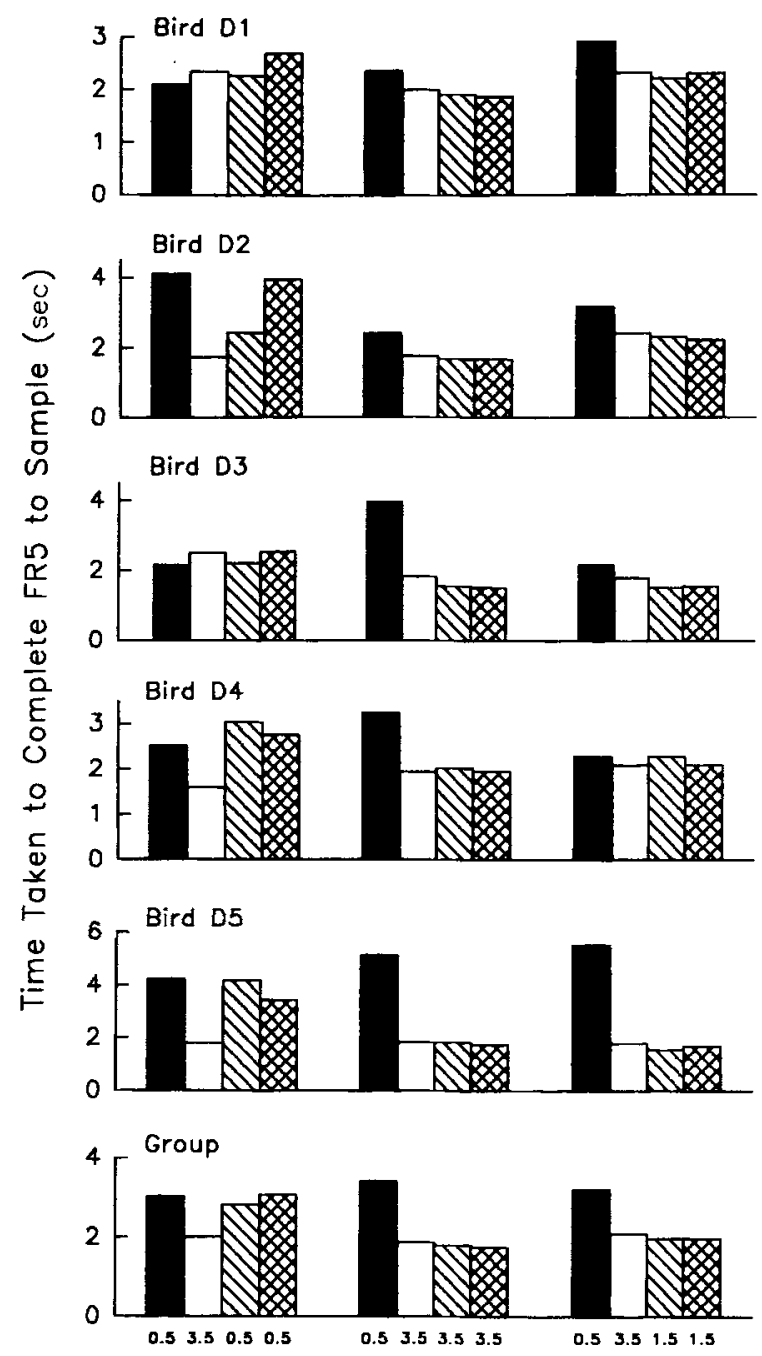

-Red Vertical $\square$-Green Vertical $\$$-Red Horiz. Green Horiz.

Figure 8. The median times taken to complete the five observing responses to each of the four sample-outcome-cue compounds in Experiment 2. The compounds were red-vertical, green-vertical, redhorizontal, and green-horizontal. In all conditions, differentoutcome trials were signaled by the vertical bar and same-outcome trials were signaled by the horizontal bar. The duration of food access for correct responses following each of the four stimulus compounds is shown on the lowest $x$-axis. The group data are the mean times of the individual birds' medians.

median times taken for a bird to complete the five responses required to each sample-outcome-cue compound from the time this stimulus appeared. These medians were calculated from the final 10 sessions of each condition. The group data are the mean response rates of the individual subjects.

Recall that in all conditions of Experiment 2 the vertical bar signaled DOs and the horizontal bar signaled SOs (i.e., in Condition 1 of Experiment 1 and all conditions in Experiment 2). In all these conditions, the com- 
bination of the vertical bar and the red sample (R-V) was followed by 0.5 -sec food access for correct responses and the combination of the vertical bar and the green sample $(\mathrm{G}-\mathrm{V})$ was followed by 3.5 -sec food access. Thus, if the magnitude of the reward affected rates of responding to the sample stimuli on these trials, and the birds were anticipating the specific reward magnitudes, then median completion times on green-sample DO trials $(G-V)$ should be shorter than times on redsample DO trials $(\mathrm{R}-\mathrm{V})$. In addition, if the duration of food access on SO trials affected rates of responding on these trials, then response rates on both SO trials ( $R-H$ and $\mathrm{G}-\mathrm{H}$ ) should not differ within conditions but should differ across conditions.

The data in Figure 8 show that the time taken to complete the sample response requirement was indeed affected by the relative magnitude of reward that was arranged on each of the four trial types. First, median times on both SO trials (R-H and $\mathrm{G}-\mathrm{H}$ ) were longer when 0.5 -sec food access was scheduled on these trials (Condition 1) than when either 1.5 or $3.5-\mathrm{sec}$ food access was scheduled (Conditions 3 and 2 , respectively). Of the 40 comparisons available, this was true in 39 cases - a difference that was significant according to a sign test $(p<$ .05). Second, consistent with outcome-expectancy theory (Peterson \& Trapold, 1982; Trapold, 1970), these data show that the birds may well have acquired differential expectancies of the rewards on DO trials. That is, there was a tendency for all birds to complete the observingresponse requirement faster on DO trials correlated with the larger reward $(\mathrm{G}-\mathrm{V})$ than on DO trials correlated with the smaller reward $(\mathrm{R}-\mathrm{V})$. This difference existed for 13 of the 15 available comparisons and was also significant according to a sign test $(p<.05)$.

Taken together, these findings suggest that the birds responded to the outcome cue and sample stimulus as a compound and learned the specific reward magnitudes associated with each. In particular, the outcome cue on both DO trials (the vertical bar) did not itself signal an overall (mean) reward magnitude on these trials. Consequently, for reasons outlined above, it is unlikely that mechanisms underlying the SME also contributed to the initial discriminability component of the DOE in the present paradigm.

\section{GENERAL DISCUSSION}

The present research examined performance characteristics of two effects of signaling the consequences for remembering in a DMTS task: the DOE and the SME. Both effects were studied within sessions by signaling the duration of food access for correct responses with outcome cues superimposed over the sample stimuli. This permitted a direct within-subject comparison of the two effects in terms of a quantitative description of DMTS performance, which employs a bias-free and unbounded estimate of stimulus discriminability. The DOE was evident as higher delayed-matching accuracies when differential magnitudes of reward were signaled for the two correct responses than when the same reward magnitude was signaled (Figures 3, 5, and 6). The SME was evident as higher delayed-matching accuracies when the same but larger reward magnitudes were signaled for both correct responses than when smaller reward magnitudes were signaled (Figure 4). However, differences between the DOE and the SME appeared when estimates of stimulus discriminability $\left(\log d_{l}\right.$, Equation 1) were plotted as a function of delay-interval duration for DO, SO, large-, and small-reward trials. A negative exponential equation (Equation 2) fitted to these data revealed differences between the initial levels and the slopes of DO and SO delay-interval functions. In contrast, the delay-interval functions describing performance on large- and small-reward trials differed only in terms of their initial levels and were similar in slope. This was confirmed by reanalyses of data provided by Nevin and Grosch (1990). In accordance with the results of previous applications of the negative exponential model (see White, 1985, 1991), these findings were interpreted as evidence that signaling DOs and signaling larger rewards enhance the initial discriminability between the samples, but only the former lowers rate of forgetting. To this end, both phenomena appear to involve time-independent or attentional processes, but only the DOE involves time-dependent or memorial processes.

Outcome-expectancy theory (Peterson \& Trapold, 1982; Trapold, 1970) described earlier provides an adequate account of why rates of forgetting were slower (i.e., estimates of $b$ were lower) on DO trials than on SO trials, and why rate of forgetting was not affected by signaling overall larger rewards. Moreover, the SME seemed reasonably explained by appealing to an attentional account (Nevin \& Grosch, 1990) where the cue signaling the larger reward may serve to enhance a subject's attention to the samples on these trials. However, given the possibility of common effects of both manipulations on estimates of initial discriminability, Experiment 2 assessed empirically the independence of these two phenomena. It examined whether the mechanism proposed to underlie the SME contributed to the enhanced initial discriminability on DO trials in Experiment 1 . The failure to find systematic effects of reward magnitude on DO and SO trial accuracies in Experiment 2 (Figures 6 and 7, and Table 3) discredited the idea that a SME contributed to the DOE in this paradigm. Furthermore, analyses of times taken to complete observing-response requirements (Figure 8 ) suggested that, in most conditions, the birds were anticipating the specific reward magnitudes on DO trials. Taken together, these results suggest that signaling DOs enhanced the initial discriminability between samples via a mechanism different from that by which signaling larger rewards enhanced initial discriminability.

The possibility that the DOE and the SME are independent phenomena is also favored by an alternative account of the mechanism underlying the initial discriminability component of the DOE. In this account, as- 
sociating DOs with the two sample stimuli enhances the discriminability between the samples by adding another stimulus dimension to the discrimination (see Edwards et al., 1982; Honig et al., 1984; Peterson \& Trapold, 1982). In contrast to the attentional account of the initial discriminability component of the DOE, this account does not predict any effect of a difference between the mean magnitude of rewards on DO trials and reward magnitudes on SO trials. According to this view, the only requirements for a DOE are that there is a discriminable difference between the rewards offered on the two DO trials and that subjects learn to anticipate the specific rewards on DO trials. Differences between the median times taken to complete the response requirement to the samples reveal that both of these criteria may well have been met in the present study. Thus, it is quite plausible that signaling DOs enhances initial discriminability by one mechanism, whereas signaling larger rewards has the same effect but by another mechanism.

To conclude, the present study joins a growing number of reports demonstrating that the rewards arranged in a DMTS task can, under some conditions, serve stimulus functions over and above whatever strengthening function they may have. We showed that there are at least two different and independent effects of signaling the consequences for remembering in a DMTS task. The DOE results when different qualities of reward, or different quantities of the same reward, are correlated with the two correct responses so that specific rewards can be anticipated following the two samples. The SME, on the other hand, results when larger rewards are signaled for both correct responses. The effect of DOs for the two correct responses was to enhance the initial discriminability between the sample stimuli and reduce the rate at which they were forgotten, whereas the effect of larger rewards was only to enhance initial discriminability. Future research may well address alternative explanations for the mechanisms underlying these two phenomena. For example, manipulation of the temporal position of the outcome cues relative to the sample stimulus and delay interval may prove useful in examining the factors contributing to the enhanced initial discriminability component of both the DOE and the SME.

\section{REFERENCES}

Berryman, R., Cumming, W. W., \& Nevin, J. A. (1963). Acquisition of delayed matching in the pigeon. Journal of the Experimental Analysis of Behavior, 6, 101-107.

BLougH, D. S. (1959). Delayed matching in the pigeon. Journal of the Experimental Analysis of Behavior, 2, 151-160.

Brodigan, D. L., \& Peterson, G. B. (1976). Two-choice discrimination performance of pigeons as a function of reward expectancy, prechoice delay, and domesticity. Animal Learning \& Behavior, 4, 121124

Carlson, J. G., \& Wielkiewicz, R. M. (1976). Mediators of the effects of magnitude of reinforcement. Learning \& Motivation, 7 , 184-196.

Cohen, L. R., Looney, T. A., Brady, J. H., \& Aucella, A. F. (1976). Differential sample response schedules in the acquisition of condi- tional discriminations by pigeons. Journal of the Experimental Analysis of Behavior, 26, 301-314.

Davison, M. C., \& Tustin, R. D. (1978). On the relation between the generalized matching law and signal detection theory. Journal of the Experimental Analysis of Behavior, 29, 331-336.

DeLong, R. E., \& Wasserman, E. A. (1981). Effects of differential reinforcement expectancies on successive matching-to-sample performance in pigeons. Journal of Experimental Psychology: Animal Behavior Processes, 7, 394-412.

Edwards, C. A., Jagielo, J. A., Zentall, T. R., \& Hogan, D. E. (1982). Acquired equivalence and distinctiveness in matching to sample by pigeons: Mediation by reinforcer specific expectancies. Journal of Experimental Psychology: Animal Behavior Processes, 7, 394-412.

EYSENCK, M. W., \& EYSENCK, C. (1982). Effects of incentive on cued recall. Quarterly Journal of Experimental Psychology, 34A, 489-498.

Ferguson, G. A. (1966). Statistical analysis in psychology and education. New York: McGraw-Hill.

Green, D. M., \& SweTS, J. A. (1966). Signal detection theory and psychophysics. New York: Wiley.

HaRnetT, P., McCarThy, D. C., \& Davison, M. C. (1984). Delayed signal detection, differential reinforcement and short-term memory in the pigeon. Journal of the Experimental Analysis of Behavior, 42, 87-111.

Hautus, M. J. (1995). Corrections for extreme proportions and their biasing effects on estimated values of $d^{\prime}$. Behavior Research Methods, Instruments, \& Computers, 27, 46-51.

Honig, W. K. (1978). Studies of working memory in the pigeon. In S. H. Hulse, H. Fowler, \& W. K. Honig (Eds.), Cognitive processes in animal behavior (pp. 211-248). Hillsdale, NJ: Erlbaum.

Honig, W. K., Matheson, W. R., \& DoDd, P. W. D. (1984). Outcome expectancies as mediators for discriminative responding. Canadian Journal of Psychology, 38, 196-217.

HoniG, W. K., \& ThOMPSON, R. K. R. (1982). Retrospective and prospective processing in animal working memory. In G. H. Bower (Ed.), The psychology of learning and motivation (Vol. 16, pp. 239282). New York: Academic Press.

Jones, B. M., \& WhITE, K. G. (1992). Sample-stimulus discriminability and sensitivity to reinforcement in delayed matching to sample. Journal of the Experimental Analysis of Behavior, 58, 159-172.

LAWRENCE, D. H. (1949). Acquired distinctiveness of cues: Transfer between discriminations on the basis of familiarity with the stimulus. Journal of Experimental Psychology, 39, 770-784.

Linwick, D., Overmier, J. B., Peterson, G. B., \& Mertens, M. (1988). Interaction of memories and expectancies as mediators of choice behavior. American Journal of Psychology, 101, 313-334.

LuCE, R. D. (1963). Detection and recognition. In R. D. Luce, R. R. Bush, \& E. Galanter (Eds.), Handbook of mathematical psychology (pp. 105-189). New York: Wiley.

MCCARTHY, D. C., \& Davison, M. C. (1984). Isobias and alloiobias functions in animal psychophysics. Journal of Experimental Psychology: Animal Behavior Processes, 10, 390-409.

MCCARThY, D. C., \& Davison, M. C. (1991). The interaction between stimulus and reinforcer control on remembering. Journal of the Experimental Analysis of Behavior, 56, 51-66.

MCCARTHy, D. C., \& NeVIN, J. A. (1991). The consequences of remembering. In W. C. Abraham, M. C. Corballis, \& K. G. White (Eds.), Memory mechanisms: A tribute to G. V. Goddard. Hillsdale, NJ: Erlbaum.

MCCARThy, D. C., \& White, K. G. (1987). Behavioral models of delayed detection and their application to the study of memory. In M. L. Commons, J. E. Mazur, J. A. Nevin, \& H. C. Rachlin (Eds.), Quantitative analyses of behavior: Vol. 5 . The effect of delay and of intervening events on reinforcement value (pp. 29-54). Hillsdale, NJ: Erlbaum.

Nelson, T. O. (1976). Reinforcement and human memory. In W. K. Estes (Ed.), Handbook of learning and cognitive processes (pp. 207246). Hillsdale, NJ: Erlbaum.

NeVIN, J. A. (1981). Psychophysics and reinforcement schedules. In M. L. Commons \& J. A. Nevin (Eds.), Quantitative analyses of be- 
havior: Vol. I. Discriminative properties of reinforcement schedules (pp. 3-27). Cambridge, MA: Ballinger.

NEVIN, J. A., \& Grosch, J. (1990), Effects of signaled reinforcer mag nitude on delayed matching-to-sample performance. Journal of Experimental Psvchology: Animal Behavior Processes, 16, 298-305.

Nevin, J. A., Mandell, C., \& Yarensky, P. (1981). Response rate and resistance to change in chained schedules. Journal of Experimental Psychology: Animal Behavior Processes, 7, 278-294.

OVermier, J. B., Bull, J. A., III, \& Trapold, M. A. (1971). Discriminative cue properties of different fears and their role in response selection in dogs. Journal of Comparative \& Physiological Psychology, 76, 478-482.

Peterson, G. B. (1984). How expectancies guide behavior. In H. L. Roitblat, T. G. Bever, \& H. S. Terrace (Eds.), Animal cognition (pp. 135-147). Hillsdale, NJ: Erlbaum.

Peterson, G. B., Linwick, D., \& Overmier, J. B. (1987). On the comparative efficacy of memories and expectancies as cues for choice behavior in pigeons. Learning \& Motivation, 18, 1-20.

Peterson, G. B., \& Trapold, M. A. (1980). Effects of altering outcome expectancies on pigeons' delayed conditional discrimination performance. Learning \& Motivation, 11, 267-288.

Peterson, G. B., \& Trapold, M. A. (1982). Expectancy mediation of concurrent conditional discriminations. American Journal of Psychology, 95, 571-580.

Peterson, G. B., Wheeler, R. L., \& Armstrong, G. D. (1978). Expectancies as mediators in the differential-reward conditional discrimination performance of pigeons. Animal Learning \& Behavior, 6, 279-285

Peterson, G. B., Wheeler, R. L., \& Trapold, M. A. (1980). Enhancement of pigeons' conditional discrimination performance by expectancies of reinforcement and nonreinforcement. Animal Learning \& Behavior, 8, 22-30.

ROBERTS, W. A. (1972). Short-term memory in the pigeon: Effects of repetition and spacing. Journal of Experimental Psychology, 94, 74-83.

SACKS, R. R., KAMIL, A. C., \& MACK, R. (1972). The effects of fixedratio sample requirements on matching to sample in the pigeon. Psychonomic Science, 26, 291-293.

SANTI, A., \& RoberTs, W. A. (1985a). Prospective representation: The effects of varied mapping of sample stimuli to comparison stimuli and differential trial outcomes on pigeons' working memory. Animal Learning \& Behavior, 13, 103-108.

SANTI, A., \& Roberts, W. A. (1985b). Reinforcement expectancy and trial spacing effects in delayed matching-to-sample by pigeons. $A n$ imal Learning \& Behavior, 13, 274-284.
Shettleworth, S., \& Nevin, J. A. (1965). Relative rate of response and relative magnitude of reinforcement in multiple schedules. Journal of the Experimental Analysis of Behavior, 8, 199-202.

SKINNER, B. F. (1938). The behavior of organisms. New York: AppletonCentury-Crofts.

Snodgrass, J. G., \& Corwin, J. (1988). Pragmatics of measuring recognition memory: Applications to dementia and amnesia. Journal of Experimental Psychology: General, 117, 34-50.

THORNDIKE, E. L. (1898). Animal intelligence: An experimental study of the associative processes in animals. Psychological Review Monograph Supplements, 2 (Whole No. 8).

Trapold, M. A. (1970). Are expectancies based upon different positive reinforcing events discriminably different? Learning \& Motivation, 1, 129-140.

Trapold, M. A., \& Overmier, J. B. (1972). The second learning process in instrumental conditioning. In A. H. Black \& W. F. Prokasy (Eds.), Classical conditioning II: Current research and theory (pp. 427-452). New York: Appleton-Century-Crofts.

URCUiOLI, P. J. (1990a). Differential outcomes and many-to-one matching: Effects of correlation with correct choice. Animal Learning \& Behavior, 18, 410-422.

UrCUIOLI, P. J. (1990b). Some relationships between outcome expectancies and sample stimuli in pigeons' delayed matching. Animal Learning \& Behavior, 18, 302-314.

UrCUIOLI, P. J. (1991). Retardation and facilitation of matching acquisition by differential outcomes. Animal Learning \& Behavior, 19, 29-36.

WhITE, K. G. (1985). Characteristics of forgetting functions. Journal of the Experimental Analysis of Behavior, 44, 15-34.

WhITE, K. G. (1991). Psychophysics of direct remembering. In M. L. Commons, J. A. Nevin, \& M. C. Davison (Eds.), Signal detection: Mechanisms, models and applications (pp. 221-237). Hillsdale, NJ: Erlbaum.

WhITE, K. G., \& McKenzIE, J. (1982). Delayed stimulus control: Recall for single and relational stimuli. Journal of the Experimental Analysis of Behavior, 38, 305-312.

Williams, D. A., Butler, M. M., \& Overmier, J. B. (1990). Expectancies of reinforcer location and quality as cues for a conditional discrimination in pigeons. Journal of Experimental Psychology: Animal Behavior Processes, 16, 3-13.

(Manuscript received July 19, 1994; revision accepted for publication September 26, 1994.) 